\title{
Experimental Demonstration of Hydrophobicity Variation in Carbon Nanotubes by Surface Modifications*
}

\author{
A. Mathur, S. Wadhwa, J. A. Byrne, S. S. Roy, ${ }^{\dagger}$ and J. A. McLaughlin \\ Nanotechnology and Integrated Bio-Engineering Centre, \\ School of Electrical and Mechanical Engineering, \\ University of Ulster, Newtownabbey, BT37 0QB, UK \\ (Received 3 June 2008; Accepted 8 October 2008; Published 4 April 2009)
}

\begin{abstract}
Surface modification of the carbon nanotubes is important for their application in various fields. In this study, multiwalled carbon nanotubes (MWCNTs) were grown on cobalt catalyst layer (3 nm thickness) sputtered on a silicon substrate using Microwave Plasma Enhanced Chemical Vapor Deposition (MPECVD) system. The as-grown MWCNT layer was found to have a thickness of $\sim 5 \mu \mathrm{m}$ and an average nanotube diameter $\sim 70 \mathrm{~nm}$. The surface of as-grown nanotubes was modified by polyvinyl alcohol (PVA). The surface hydrophobicity has been estimated by measuring the static contact angles of water on nanotube surfaces before and after the modification by varying PVA concentration in the range of 1:10,000-5:10,000 (w/v). This functionalisation showed a dramatic change of the surface of nanotubes from highly hydrophobic to hydrophilic. The fictionalization of MWCNTs was confirmed further with electrochemical measurements.

[DOI: $10.1380 /$ ejssnt.2009.334]
\end{abstract}

Keywords: Nanotubes; Scanning electron microscopy (SEM); Sputtering; Contact angle; Surface energy

\section{INTRODUCTION}

Multiwalled carbon nanotubes (MWCNTs) and singlewalled carbon nanotubes (SWCNTs) have attracted great scientific attention because of their exceptional physical and chemical properties. Hydrophilic surfaces of CNTs have huge potential for their application in chemical and biological sensing devices. The enhancement in the electrocatalytic activity of CNTs due to their one dimensional structure has been reported $[1,2]$.

For the functionalisation and tuning the physical properties of CNTs, various polymers have been introduced in CNTs network and vice versa. In recent years PVA and CNTs composites have been reported. The PVA is a water-soluble, biologically compatible, nontoxic, and readily available low-cost synthetic polymer. It has good forming properties and impact strength. After a freezingthawing process, the PVA chains are physically crosslinked by semipermanent entanglement, molecular association, or crystallites $[3,4]$. The PVA is hydrophilic in nature and consists of an $\mathrm{OH}$ - group at every other carbon in the chain and hence crosslinkable. The interfacial between polymer and CNTs are of great interest in various applications.

In this paper, multiwalled carbon nanotubes (MWCNTs) were grown on cobalt catalyst layer. The surface of as-grown nanotubes was modified by polyvinyl alcohol (PVA) and the wettability of the nanotubes was investigated by evaluating their contact angle using sessile drop method. The electrical impedance spectroscopy was carried out on these modified nanotubes by using Autolab.

\footnotetext{
* This paper was presented at the 14th International Conference on Solid Films and Surfaces (ICSFS-14), Trinity College Dublin, Ireland, 29 June - 4 July, 2008.

†Corresponding author: s.roy@nibec.ulster.ac.uk
}

\section{EXPERIMENTAL DETAILS}

Multiwalled carbon nanotubes (MWCNTs) were grown on cobalt catalyst layer (3 nm thickness) sputtered on a silicon substrate using microwave plasma enhanced chemical vapor deposition (MPECVD) system using methane and nitrogen. The catalyst for all the samples was deposited by using sputter coater system. The nanotubes were functionalised using aqueous solution of $\mathrm{PVOH}$ (100\% hydrolyzed, Average MW-14K, Aldrich Chemicals), which was prepared by mixing PVOH with deionized water in the range of $1: 10,000$ to $5: 10,000(\mathrm{w} / \mathrm{v})$ and heating at $70^{\circ} \mathrm{C}$ for 2 hour with constant stirring.

The substrate with MWCNTs was immersed in the solution overnight, since the functionalisation of $\mathrm{PVOH}$ occurs while cooling. The substrates were then removed by rinsing with copious amount of de-ionized water and drying under vacuum. To study the effect on the wettability of the carbon nanotubes by $\mathrm{PVOH}$ functionalisation, dynamic contact angles were measured by using contact angle analyzer. The contact angles of the functionalized CNTs were compared with that of the pristine carbon nanotubes. The electrical characterization was carried out by using electrochemical impedance spectroscopy (EIS) using Autolab.

\section{RESULTS AND DISCUSSION}

The SEM micrographs of multiwalled carbon nanotubes before and after functionalisation with $\mathrm{PVOH}$ are illustrated in Fig. 1 and Fig. 2 respectively. Comparing the two images, it was evident that even a small amount $(0.0004 \% \mathrm{w} / \mathrm{v})$ of PVOH can significantly change the surface morphology of the nanotubes. Recently it has been reported that CNTs generate crystallization of PVA and crystalline layer may be formed around the nanotube surface [10-12]. The Fig. 2 also indicates a strong interfacial 


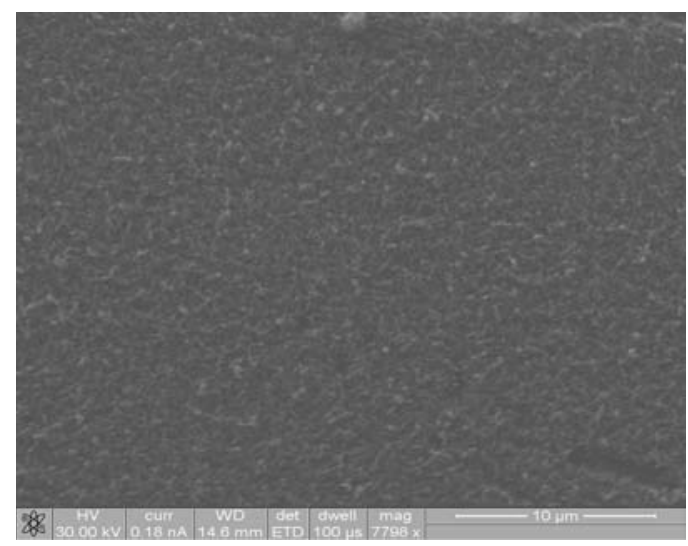

FIG. 1: MWCNT before functionalisation.

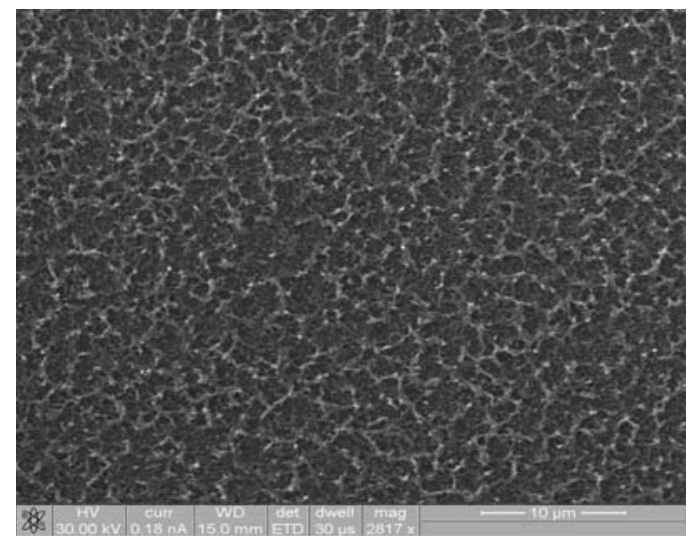

FIG. 2: MWCNT after functionalisation.

bonding between PVA and MWCNTs.

The static contact angle measurements were performed using the sessile drop method, the water droplets $(2 \mu \mathrm{L})$ were deposited using a micro-syringe and images were recorded to evaluate the angle of the liquid-solid interface on the both sides (Left (L) and Right (R)) of the droplet. Two optical images of the water droplet deposited on the multiwalled nanotubes before and after functionalisation are shown in Figs. 3(a) and 3(b) respectively. The effect of $\mathrm{PVOH}$ functionalisation was quite evident from the two images and revealed a transformation of hydrophobic surface to hydrophilic surface. The PVOH tends to crystallize and introduce an alcohol functional group on the surface of the nanotubes. The basic mechanism of interaction between the PVOH and nanotubes can be attributed to the lowering of interfacial free energy which is evident from the change in the contact angles of the functionalized MWCNTs [13].

The change in the wettability of the MWCNTS surface by varying PVOH concentration was further studied by the static contact angle measurement. The results are listed in Table I. As the PVOH concentration was increased the measured contact angle decreased. In Table I it can be seen that there is a maximum change in contact angle from 111 to 28 corresponding to an average of $74 \%$ decrease when carbon nanotubes are functionalized.

In general the surface of the nanotubes is covered by
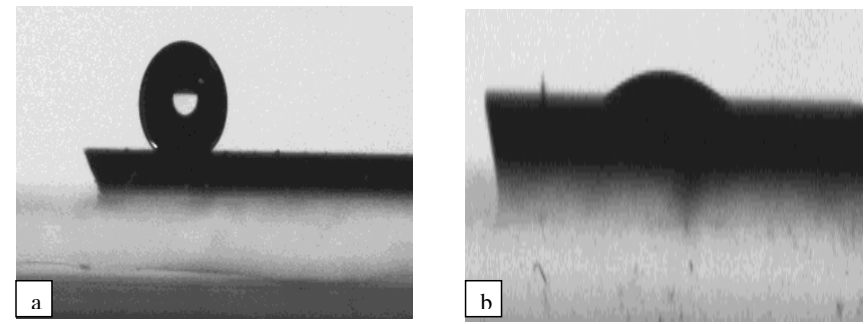

FIG. 3: Effect on the water droplet deposited on the multiwall carbon nanotubes. (a) Before and (b) after functionalization.

TABLE I: Contact angle variation with respect to the PVOH concentration.

\begin{tabular}{ccc}
\hline \hline Sample & Theta (L) & Theta (R) \\
\hline MWCNT & 111.28 & 110.55 \\
5:10,000 MWCNT+PVOH & 28.02 & 22.51 \\
4:10,000 MWCNT+PVOH & 37.71 & 36.03 \\
3:10,000 MWCNT+PVOH & 39.05 & 38.82 \\
2:10,000 MWCNT+PVOH & 40.1 & 40.92 \\
1:10,000 MWCNT+PVOH & 53.22 & 47.06 \\
\hline \hline
\end{tabular}

active $\mathrm{C}$ atoms and $\mathrm{C}-\mathrm{C}$ which are covalently bonded and carbon dangling bonds. The introduction of $\mathrm{PVOH}$ on MWCNTs may have two effects: firstly $\mathrm{OH}-$ may reacts with the active $\mathrm{C}$ or the dangling bonds and form a strong interfacial bond. Alternatively, nanotubes were coated by thin layer of crystalline or amorphous PVOH. Higher concentration of $\mathrm{PVOH}$ have a much higher tendency to react with the surface walls of the $\mathrm{CNT}$, hence lower the interfacial free energy. The above results are consistent with those reported by Vedala et al. [13].

Cyclic voltammetry is a common and useful electrochemical procedure used to test the properties of electrodes or solutions. Cyclic voltammograms and impedance spectra were measured with an autolab Electrochemical Analyzer. The cyclic voltammogramms were obtained using $1.0 \mathrm{mM}$ potassium ferricyanide $\left(\mathrm{K}_{3} \mathrm{Fe}(\mathrm{CN})_{6}^{3-}\right)$ in $0.1 \mathrm{M}$ potassium chloride $(\mathrm{KCl})$ aqueous solution as the supporting electrolyte at a scan rate of $100 \mathrm{mV} / \mathrm{s}$ in the frequency range $10 \mathrm{mHz}-10 \mathrm{MHz}$.

The cyclic voltammogram of MWCNT before and after functionalisation with PVOH is shown in Fig. 4. The oxidation peak of MWCNT appeared at $288 \mathrm{mV}$ and shifted to $469 \mathrm{mV}$ in the positive direction after functionalization with $\mathrm{PVOH}$. The redox potential difference within MWCNT functionalized with PVOH was larger $(334 \mathrm{mV})$ compared to that of MWCNT $(162 \mathrm{mV})$. The separation between the oxidation and reduction peak potentials $\Delta E_{\mathrm{p}}$ of $\left[\mathrm{Fe}(\mathrm{CN})_{6}\right]^{-3}$ for PVOH modified CNTs was higher than that of untreated CNTs, which indicates a slow electrode kinetics in modified CNTs. Also the difference between the areas of both the curves suggests the capacitive behavior of MWCNT functionalized with PVOH. 


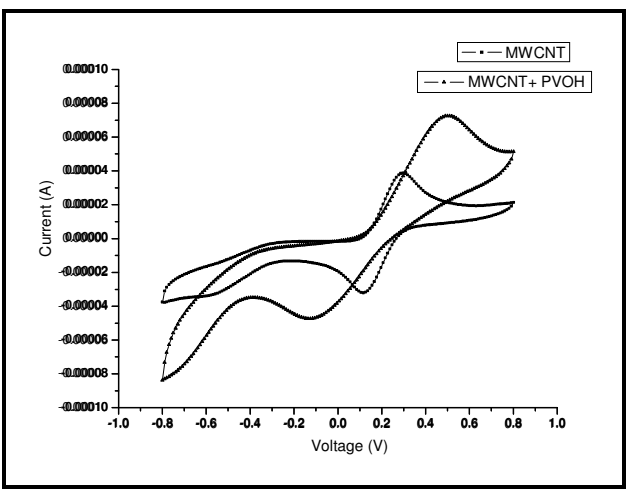

FIG. 4: Cyclic voltammogram of MWCNT before and after functionalisation with PVOH.

\section{CONCLUSION}

In this study multiwall carbon nanotubes were functionalized by using $\mathrm{PVOH}$. It was shown that $\mathrm{PVOH}$ is able to modify the surface behavior of multiwall carbon nanotube from hydrophobic to highly hydrophilic. These findings are being further considered for the possible use of functionalized carbon nanotubes for humidity, gas and bimolecular sensing. The electrical characterization is also being studied to understand the charge transport mechanism in these modified carbon nanotubes. The anodic, cathodic peak separation increased in $\mathrm{PVOH}$ modified MWCNTs, indicating lower electron transfer kinetics. The MWCNT and PVOH composites can be directly integrated with microelectronics and microfluidic devices for various purposes [13].
[1] C. E. Banks, R. R. Moore, T. J. Davies, and R. G. Compton, Chem. Commun. 16, 1804 (2004).

[2] R. R. Moore, C. E. Banks, and R. G. Compton, Anal. Chem. 76, 2677 (2004).

[3] S. R. Stauffer and N. A. Peppas, Polymer 33, 3932 (1992).

[4] K. Y. Lee, D. J. Mooney, Chem. Rev. 101, 1869 (2001).

[5] D. E. Packham, Int. J. Adhesion Adhesives 23, 437 (2003).

[6] G. D. Christian, Analytical Chemistry (John Wiley \& Sons, New York, 1980), p. 346.

[7] H. Peng, C. Soeller, N. A. Vigar, P. A. Kilmartin, and J. T. Sejdic, biosensors and bioelectronics 20, 1821 (2005).

[8] H. Peng, C. Soeller, N. A. Vigar, V. Caprio, and J. T.
Sejdic, biosensors and bioelectronics 22, 1868 (2007).

[9] M. Cadek, J. N. Coleman, K. Hedicke, and W. J. Blau, Appl Phys. Lett. 83, 2718 (2003).

[10] M. Cadek, J. N. Coleman, V. Barron, K. Hedicke, and W. J. Blau, Appl. Phys. Lett. 81, 5123 (2002).

[11] X. Zhang, T. Liu, T. V. Sreekumar, S. Kumar, V. C. Moore, R. H. Hauge. and R. E. Smalley, Nano Lett. 3, 1285 (2003).

[12] W. Chen, X. Tao, P. Xue, and X. Cheng, Appl. Surf. Sci. 252, 1404 (2005).

[13] H. Vedala, X. Y. Zhou, and G. Kim, Appl. Surf. Sci. 252, 7987 (2006). 